\title{
THE ROLE OF ELLIPTICITY AND NORMALITY ASSUMPTIONS IN FORMULATING LIVE-BOUNDARY CONDITIONS IN ELASTICITY*
}

\author{
BY \\ P. PODIO - GUIDUGLI (Universitá di Roma-Tor Vergata) \\ AND \\ G. VERGARA CAFFARELLI AND E. G. VIRGA (Universitúdi Pisa)
}

1. Introduction. The mechanical interaction of a body with its environment is a varied and complex phenomenon which is difficult both to observe and to describe carefully. Boundary conditions modeling nontrivial interactions are difficult to formulate mathematically; besides, they may easily lead to unusual boundary value problems, perhaps even so unusual as to challenge and defeat an expert analyst. Not only that, blunt generalization of the few well-understood types of boundary conditions may pose rather subtle problems of interpretation and classification to the expert mechanist.

In Sec. 2, the bulk of this paper, we develop a train of reasoning that leads us to formulate an unambiguous boundary condition of traction, reproducing the nonlinear interaction of an elastic body and an elastic environment in strict contact with it; the emphasis here, in accordance with our title, is on the role played by ellipticity and normality hypotheses.

In Sec. 3 we briefly discuss the importance of ellipticity and normality in problems of elastostatics linearized about a stressed equilibrium placement. In particular, we show by means of an explicit example that, if the environment is live but normality fails to hold, one can easily run into taxonomic troubles.

2. The Genuine Live-Boundary Condition of Traction. Let a (possibly unbounded) open regular region $\mathscr{B}$ of $R^{M}$, with $M=2$ or 3 , be separated from its exterior $\mathscr{E}$ by a smooth orientable surface $\mathscr{S}$, the common boundary of $\mathscr{B}$ and $\mathscr{E}$, with $n$ the exterior unit normal to $\mathscr{B}$.

Once constitutive prescriptions have been given, we may think of $\mathscr{B}$ as the body and of $\mathscr{E}$ as the environment of the body $\mathscr{B} .{ }^{1}$ Then, given any judiciously chosen set of data, we may formulate a variety of mechanically significant problems, any one of which ultimately

\footnotetext{
* Received January 21, 1985.

${ }^{1}$ Notice that here we implicitly choose not to consider the interesting case when $\mathscr{S}$ itself is thought of as a material surface (in the manner, e.g., of Gurtin and Murdoch [1]) and therefore made the object of a peculiar constitutive prescription. To contemplate such a case would require a more delicate analysis.
} 
reduces to determining the state (i.e., the triplet of displacement, strain, and stress) over $\mathscr{B} \cup \mathscr{E}$, provided a suitable set of jump conditions has been stipulated for the state at the points of $\mathscr{S}$.

Loosely, our goal is to sketch a credible scenario where a large class of such problems for $\mathscr{B} \cup \mathscr{E}$ can be reduced through a rational procedure to a corresponding problem for $\overline{\mathscr{B}}=\mathscr{B} \cup \mathscr{S}$, with the presence of $\mathscr{E}$ replaced by a set of bounciary conditions on $\mathscr{S}$. To anticipate one of our main findings, perhaps not about to come as any great surprise to the knowledgeable reader, these boundary conditions will as a rule be of the live type; i.e., they will be prescriptions of the surfacial stress vector which depend functionally in a nontrivial way on the solution in $\overline{\mathscr{B}}$.

Now let $u, T$ denote the restrictions to $\mathscr{S}$ of the unique extensions $\dot{u}, \dot{T}$ to $\overline{\mathscr{B}}$ of smooth displacement and stress fields $\stackrel{\leftrightarrow}{u}, \stackrel{\circ}{T}$ over $\mathscr{B}$; further, let $(u, t)$, with $t=T n$, denote the pair of fields over $\mathscr{S}$ consisting of the displacement $u$ and the stress vector $t$; finally, let $(v, s)$ be defined over $\mathscr{S}$ in a completely analogous way, but starting from displacement and stress fields $\stackrel{\circ}{v}$ and $\stackrel{\circ}{S}$ defined over $\mathscr{E}$. We wish to interpret $v(x), s(x)$ as, respectively, the displacement of boundary point $x$ of the environment and the contact force exerted at $x$ by the environment over the body.

We are interested in contact forces that depend functionally on the displacement field over $\mathscr{E} \cup \mathscr{S}$. Suppose that the stress response of $\mathscr{E}$ to deformation has a local character, as is the case for an elastic environment. Then, for $x \in \mathscr{S}$ fixed, and for any given field $\dot{v}$ on $\mathscr{E}$, we tentatively choose the functional dependence of $s$ on $\dot{v}$ as follows:

$$
s(x)=\hat{\mathfrak{f}}(v(x), D \dot{v}(x)),
$$

where $D$ is the gradient operator.

Let us now decompose the gradient operator $D$, at a point $x \in \mathscr{S}$, into its normal and tangential parts $D_{n}$ and $D_{t}$ :

$$
D \dot{v}(x)=D_{n} \dot{v}(x)+D_{t} \dot{v}(x)
$$

with

$$
D_{n} \dot{v}(x)=\partial_{n} \dot{v}(x) \otimes n(x), \quad D_{t} \dot{v}(x)=D_{t} v(x),
$$

and with $\partial_{n}$ denoting the operation of differentiating along the direction $n(x)$, the normal to $\mathscr{S}$ at $x$. Then, in view of $(2.1)-(2.3)$, we replace $(2.1)$ by

$$
s(x)=\mathfrak{f}\left(v(x), D_{t} v(x), \partial_{n} \dot{v}(x)\right) .
$$

Our next step is to lay down a set of jump conditions for the state at the points of $\mathscr{S}$. We say that the body $\mathscr{B}$ and its environment $\mathscr{E}$ are in strict contact in a neighborhood oi a point $x$ of their surface of separation $\mathscr{S}$ if

$$
u(x)=v(x) \text { and } t(x)=s(x) .
$$

In strict contact, both the displacement and its tangential gradient suffer no jump at $\mathscr{S}$; besides, the continuity of the stress vector at $\mathscr{S}$ generally implies restrictions on the jump condition for the normal derivative of displacement. To motivate the ad hoc assumptions we shall make to determine those restrictions, we turn momentarily to linear elasticity. 
Suppose that $\mathscr{B}$ and $\mathscr{E}$, besides being in strict contact, are made up of two linearly elastic materials with elliptic elasticity tensors $\mathbb{C}$ and $\mathbb{D}$, respectively, so that $\mathbb{C}$, say, obeys

$$
\operatorname{det} \mathbb{C}^{0}(a) \neq 0 \quad \text { for } C_{i h}^{0}(a)=C_{i j h k} a_{j} a_{k}, a \neq 0 \text {. }
$$

If $\mathbb{C}(\mathbb{D})$ can be first extended and then restricted to $\mathscr{S}$ in such a way as to preserve ellipticity, the matrix field

$$
A=C^{0}(n) \quad\left(B=D^{0}(n)\right)
$$

has invertible values on $\mathscr{S}$. Thus, as

$$
t=(\mathbb{C} D \dot{u}) n=A \partial_{n} \dot{u}+\left(\mathbb{C} D_{t} u\right) n,
$$

we have that

$$
\partial_{n} \dot{u}=A^{-1}\left(t-\left(\mathbb{C} D_{t} u\right) n\right),
$$

a formula which shows that, once $u$ is assigned on $\mathscr{S}, t$ and $\partial_{n} \dot{u}$ are essentially interchangeable bits of information at a point of $\mathscr{S}$. Likewise,

$$
\partial_{n} \dot{v}=B^{-1}\left(s-\left(\mathbb{D} D_{t} u\right) n\right) .
$$

Thus, in particular, if $\mathscr{B}$ and $\mathscr{E}$ are in strict contact, linearly elastic, and elliptic, the normal derivative of displacement suffers at $\mathscr{S}$ a discontinuity depending on the local values of the stress vector and the tangential gradient of displacement, as $(A, \mathbb{C})$ and $(B, \mathbb{D})$ may be expected, in general, to differ.

REMARK 1. It is important to note that ellipticity, and not linearity of the constitutive law, is the crucial assumption. Indeed, for $\mathscr{B}$ composed of a nonlinearly elastic material, (7) 2 is replaced by

$$
t=T\left(\partial_{n} \dot{u} \otimes n+D_{t} u\right) n,
$$

and this equation is locally solvable for $\partial_{n} \dot{u}$ if the ellipticity condition prevails, i.e.,

$$
\operatorname{det} C(a) \neq 0 \quad \text { for } C_{i h}(a)=\mathbb{T}_{i j h k} a_{j} a_{k}, a \neq 0, \mathbb{T}=\frac{\partial T}{\partial(D \dot{u})} .
$$

REMARK 2. For boundary value problems ruled by PDEs, ellipticity hypotheses typically involve the coefficients of the field operator; here, (EC) plays an important role at the boundary.

In light of the above, if one assumes that $\mathscr{B}$ and $\mathscr{E}$ are in strict contact, then (2.5) allows one to give (2.4) the following provisional form:

$$
s(x)=\mathfrak{f}\left(u(x), D_{t} u(x), \partial_{n} \dot{v}(x)\right) .
$$

Moreover, for elliptic elastic pairs of a body $\mathscr{B}$ and an environment $\mathscr{E}$, we make the following constitutive assumption: that both $\partial_{n} \dot{u}$ and $\partial_{n} \dot{v}$ depend on the tangential gradient and the stress vector

$$
\partial_{n} \dot{u}=u\left(D_{t} u, t\right), \quad \partial_{n} \dot{v}=\mathfrak{v}\left(D_{t} u, s\right),
$$

and that the dependence on the stress vector is essential, in that the matrices $\partial \mathfrak{u} / \partial t$ and $\partial \mathfrak{b} / \partial s$ are invertible. Thus, by (2.11) and (2.12), there exists a function $\mathfrak{g}$ such that

$$
s(x)=\mathfrak{g}\left(u(x), D_{t} u(x), s(x)\right) .
$$


As a last step toward our goal, we assume that the condition (2.13) is genuine, in that for no assignment of $u, D_{t} u$ does it reduce to an identity in $s$. Accordingly, we require that

$$
\operatorname{det} L \neq 0, \text { with } L=1-\frac{\partial g}{\partial s} .
$$

(GC) implies that (2.13) is locally solvable for $s$; then $s$ can be expressed as a function of $u$ and $D_{t} u$ :

$$
s(x)=s\left(u(x), D_{t} u(x)\right) \text {. }
$$

The last equation suggests that in formulating boundary value problems the contact force exerted by the environment on the body should generally be chosen of the live type.

REMARK 3. The special dependence (2.14) of $s$ on boundary information concerning the solution has been considered by Spector $[2,3]$, who has termed simple such surfacial loading operators. Spector's choice was motivated by the observation, due to Gurtin [4], that pressure loading, a well-known example of live loading, is indeed simple.

REMARK 4. For two linearly elastic elliptic bodies in strict contact, (2.11) takes the form

$$
s=B \partial_{n} \dot{v}+\left(\mathbb{D} D_{t} u\right) n
$$

and is readily inverted as

$$
\partial_{n} \dot{v}=B^{-1}(s-(\mathbb{D} D, u) n),
$$

which in turn corresponds to $(2.12)_{2}$. Therefore, (2.13) reduces to an identity, and (GC) cannot be satisfied. This indicates that, in general, it is not sound to require (GC) in a contact situation. However, on passing from contact to boundary conditions, we believe that one can regard $(2.12)_{2}$ as a separate constitutive assumption, independent of (2.11) and consistent with it in the peculiar sense made explicit by (GC).

Substitution of (2.14) into (2.12) 2 and, with use made of $(2.5)_{2}$, into $(2.12)_{1}$ allows us to complete, with a suitable jump condition for the normal derivative of displacement, the set of jump conditions for the state to be stipulated at a point of $\mathscr{S}$. In summary, under hypotheses of strict contact, ellipticity, and genuinity, the displacement $u$ and its tangential gradient $D_{t} u$ must be continuous, whereas the normal derivative of displacement may suffer a jump described by a function of $u$ and $D_{t} u$, whose choice has constitutive character.

We are now in a position to state a boundary condition of traction which would describe a large class of contact actions exerted by an environment of elastic type on an elastic body.

Naively, one might write a live-boundary condition of traction for $\mathscr{B}$ as

$$
t(x)=s(x) \text { for } x \in \mathscr{S} .
$$

or rather [see (2.1) and (2.10)], as

$$
\overline{\mathfrak{l}}\left(u, D_{t} u, \partial_{n} \dot{u}\right)(x)=0 \text { for } x \in \mathscr{S},
$$

with the boundary operator $\bar{l}$ defined by

$$
\bar{l}\left(u, D_{t} u, \partial_{n} \dot{u}\right)=T\left(\partial_{n} \dot{u} \otimes n+D_{t} u\right) n-\hat{\mathfrak{i}}\left(u, \partial_{n} \dot{u} \otimes n+D_{t} u\right) .
$$


On the other hand, in view of $(2.5),(2.12)_{1}$ and (2.14), the boundary operator should have the form

$$
\mathfrak{l}\left(u, D_{t} u, \partial_{n} \dot{u}\right)=\partial_{n} \dot{u}-\bar{u}\left(u, D_{t} u\right) .
$$

Now $\mathfrak{I}$ is locally reducible to $\mathfrak{I}$ if the implicit equation

$$
\overline{\mathfrak{I}}\left(u, D_{t} u, \partial_{n} \dot{u}\right)=0
$$

can be locally solved for $\partial_{n} \dot{u}$; this is the case if the following normality condition holds:

$$
\operatorname{det} M(n) \neq 0 \quad \text { for } M(n)=C(n)-\mathrm{ff} n, \mathrm{ff}=\frac{\partial \hat{\hat{\varphi}}}{\partial(D \dot{u})} \text {. }
$$

Within the scenario we have set up, accepting (NC) is mandatory. We observe that (EC) implies (NC) when the environment is dead, and $\hat{f}$ reduces to an assigned function of $x$ only over $\mathscr{S}$; more generally, when $\hat{f}$ is independent of the deformation gradient; and finally, when $\hat{f}$ is simple in the sense of Remark 3.

We call (2.15), with $\overline{\mathfrak{l}}$ restricted by (NC), the genuine live-boundary condition of traction.

3. Ellipticity and Normality in Linearized Elastostatics. When one passes from a local study of a nonlinear problem to the corresponding linearized problem, both (EC) and (NC) continue to play an important role. Indeed, our present (NC) is a version appropriate to elasticity of the homonymous condition introduced by Geymonat [5] and Grubb [6] in the general theory of linear elliptic systems, and appears to be the natural extension to systems of the normality condition for scalar operators (e.g., see [7], Chapter 2).

If (EC) and (NC) prevail, we have shown in [8] that a Green's formula, which generalizes the reciprocity formula of Betti of classical elasticity, holds for the traction boundary value problem of linearized elastostatics with live loads. Such a Green's formula allows one (i) to define formal adjoints to both the field and boundary operators, (ii) to determine a set of conditions sufficient for self-adjointness, and (iii) to state compatibility conditions on the data necessary and sufficient [9] for solvability of the underlying boundary value problem in a familiar Hilbert space setting.

We now wish to give an example of the ambiguities that may accompany the failure of (NC).

In view of (2.15), a linearized live-boundary condition of traction can be written as

$$
(T D \dot{u}(x)) n(x)-\text { ff } D \dot{u}(x)-F u(x)=0,
$$

where $u$, with slight abuse of notation, is now used for the first approximation of the displacement from the reference placement:

$$
\begin{gathered}
t(x)=(T D \dot{u}(x)) n(x), \\
s(x)=\mathrm{f} D \dot{u}(x)+F u(x), \quad \text { with } F=\frac{\partial \hat{f}}{\partial u} .
\end{gathered}
$$

Choose $\hat{f}$ to describe a live environment such that

$$
f_{i h k}=P_{i l} \pi_{l j h k} n_{j}, \quad F_{i h}=-P_{i h},
$$


with $P$ a perpendicular projection field over $\mathscr{S}$. Then it is easy to check that the matrix $M(n)$, which appears in the statement of (NC), reduces to

$$
M(n)=(1-P) C(n)
$$

and is, therefore, singular, whereas (3.1) takes the form

$$
(1-P) t+P u=0,
$$

or, equivalently,

$$
(1-P) t=0 \text { and } P u=0 .
$$

One promptly recognizes in (3.7) a generalized boundary condition of the dead type (see [10], Chapter V). In particular, when $P=n \otimes n,(3.7)$ reduces to the so-called contact boundary condition:

$$
t-(t \cdot n) n=0 \text { and } u \cdot n=0 .
$$

Thus, for $\mathrm{ff}, F$ as in (3.4) and $P=n \otimes n$, the boundary condition (3.1) can be equally well classified as a live-boundary condition of traction or as a dead-boundary condition of contact.

Other similar examples can be concocted. But the validity of (NC) precludes any pathology.

Acknowledgment. We acknowledge a stimulating discussion with G. Geymonat. We also thank W. Williams and M. E. Gurtin for many useful comments.

\section{REFERENCES}

[1] M. E. Gurtin and A. I. Murdoch, A continuum theory of elastic material surfaces, Arch. Rat. Mech. Anal. 57, 291-323 (1975)

[2] S. J. Spector, On uniqueness in finite elasticity with general loading, J. Elasticity 10, 145-161 (1980)

[3] S. J. Spector, On uniqueness for the traction problem in finite elasticity, J. Elasticity 12, 367-383 (1982)

[4] M. E. Gurtin, On the non-linear theory of elasticity, in Contemporary developments in continuum mechanics and partial differential equations (G. M. de La Penha and L. A. J. Medeiros, eds., pp. 237-253, North Holland, Amsterdam (1978)

[5] G. Geymonat, Su alcuni problemi ai limiti per i sistemi lineari ellittici secondo Petrowskii, Matematiche 20 , 211-253 (1965)

[6] G. Grubb, Weakly semibounded boundary problems and sesquilinear forms, Ann. Inst. Fourier Grenoble 23, 145-194 (1973)

[7] J. L. Lions and E. Magenes, Problèmes aux limites non homogènes, Dunod, Paris (1968)

[8] P. Podio-Guidugli, G. Vergara Caffarelli and E. G. Virga, Una formula di Green per il problema di carichi vivi dell'elastostatica linearizzata, Atti VII Congr. AIMETA, Trieste, vol. 2, pp. 1-10 (2-5 October 1984)

[9] P. Podio-Guidugli, G. Vergara Caffarelli and E. G. Virga, in preparation,

[10] M. E. Gurtin, The linear theory of elasticity, in Handbuch der physik, vol. VIa/2 (C. Truesdell, ed.), Springer, Berlin (1972) 